Apidologie, 1981, $12(3), 269-288$.

\title{
EFFET DE L'ACIDE CÉTO-9 DECENE-2 TRANS OÏQUE (PHÉROMONE DE LA REINE D'ABEILLE, APIS MELLIFICA L.) SUR LE TAUX DE MORTALITÉ D'OUVRIÈRES ENGAGÉES
}

\author{
Ghada NAHAS-ZAHREDDINE*, Janine PAIN**
}

\begin{abstract}
RÉSUMÉ
Après avoir mis en évidence (Roger, PAIN, 1966) l'influence bénéfique de reines d'abeilles sur le taux de mortalité des ouvrières accompagnatrices, les auteurs étudient l'effet de la phéromone royale (acide céto-9 décène- 2 oïque de synthèse) sur la mortalité de petits groupes d'ouvrières (50) âgées de 1 à 3 jours. La phéromone en solution alcoolique est présentée sur des languettes de papier. Les doses utilisées sont de $25,50,75,100,200 \mu \mathrm{g}$ d'acide/cagette/jour pendant 3 à 4 semaines. Chaque série expérimentale comprend 10 cagettes.

L'analyse des courbes de mortalité met en évidence que c'est la dose de $25 \mu \mathrm{g}$ d'acide qui augmente de façon significative la durée de vie des ouvrières. La dose de $200 \mu \mathrm{g}$ présente également une action favorable mais elle est moins efficace. Les doses intermédiaires sont sans action.
\end{abstract}

On discute l'hypothèse de deux mécanismes d'action.

\section{INTRODUCTION}

La reine d'abeilles émet des phéromones qui sont localisées au niveau des glandes tergales (RENNER, VIERLING, 1977) et des glandes mandibulaires (BUTLER, SIMPSON, 1958 et PaIN, 1961). Ces dernières sécrètent de nombreuses substances parmi lesquelles on trouve deux acides phéromonaux. Il s'agit des acides céto-9 et hydroxy-9 décène-2 trans oïque. De nombreuses équipes tant en France (BARBIER et coll., 1960) qu'à l'étranger ont effectué des synthèses de l'acide céto-9 décène- 2 oïque (CALLOw, JoHNSTON, 1960).

* Université libanaise, faculté des Sciences, Hadeth Beyrouth (Liban). (France).

** Station de Recherches sur l'Abeille et les Insectes sociaux, I.N.R.A. 91440 Bures-sur-Yvette 
Les actions qu'exercent les deux phéromones de synthèse sur les ouvrières accompagnatrices sont multiples et se répercutent à la fois sur le comportement (attraction sexuelle pour les mâles d'abeilles, attraction d'agrégation pour les ouvrières essaimantes - stimulation du comportement de butinage - inhibition de la construction des cellules royales) et la physiologie des ouvrières réceptrices (inhibition du développement des ovaires).

La reine vivante influence d'autres processus tels que la prise de nourriture sucrée et la durée de vie d'ouvrières claustrées lesquelles se trouvent améliorées de façon significative (Roger, 1971 et Roger, Pain, 1966).

Étant donné que les reines contiennent de l'acide céto-9 décène- 2 oïque dans la tête et sur le corps, on s'est demandé si cet acide ne serait pas responsable en partie de la diminution de la mortalité de petits groupes d'ouvrières d'age connu. C'est le travail de RoGER, PAIN, 1966 qui a été repris, en offrant aux ouvrières, non plus des reines vivantes ou mortes mais des papiers imprégnés de l'une des phéromones de synthèse : l'acide céto- 9 décène- 2 oïque.

\section{MATÉRIELS ET MÉTHODE}

\section{MATÉRIEL BIOLOGIQUE}

a) Les ouvrières

Elles proviennent de cadres de couvain naissant à l'étuve à $32^{\circ} \mathrm{C} \pm 1{ }^{\circ} \mathrm{C}$. Le rucher couvert a fourni les cadres de couvain en mars (expérience 1). Au printemps, les jeunes abeilles sont obtenues à partir de cadres de couvain naissant prélevés dans les colonies installées dans la nature. Nous n'avons jamais utilisé d'abeilles naissantes sur un cadre de couvain ayant séjourné plus de 4 jours à l'étuve. Pour obtenir un nombre suffisant d'ouvrières à encager, nous avons prélevé des abeilles âgées de 1 à 3 jours. Pour une meilleure répartition des abeilles écloses, nous les avons introduites par groupes de 10 dans chacune des cagettes constituant une série expérimentale jusqu'à l'obtention de 50 individus par cagette.

\section{b) Les reines de substitution}

Ce sont des leurres de papier utilisés en chromatographie (papier d'Arches). Ils sont préparés sous forme de languettes de $15 \mathrm{~mm}$ de large sur $90 \mathrm{~mm}$ de long. Les languettes sont pliées en deux dans le sens de la longueur pour faciliter leur introduction dans l'un des orifices du toit de la cagette. Les leurres sont imprégnés de $0,1 \mathrm{ml}$ d'éthanol à $95^{\circ}$ contenant une quantité connue d'acide céto- 9 décène- 2 oïque de synthèse*.

\section{MATÉRIEL TECHNIQUE}

Les cagettes expérimentales que nous avons utilisées sont du type PaIN (1966), modèle mis au point par la Station de Recherches sur l'Abeille et les Insectes Sociaux à Bures-sur-Yvette. Signalons que le couvercle transparent est percé de 2 orifices par lesquels on introduit un abreuvoir rempli d'eau et dans notre cas, un leurre qui est maintenu par un bouchon de liège. Elles sont munies d'une lame de cire gaufrée collée

* L'acide céto-9 décène-2 oïque provient des laboratoires pharmaceutiques suivants : Sefton Bulk Pharmaceuticals Ltd. Greenford Road, Greenford, Middlexex, England, UB 6 OHE. 
au toit et de deux mangeoires contenant l'une la nourriture sucrée (candi, mélange de miel et de sucre glace en proportion $1: 3$ ), l'autre la nourriture azotée (pollen en pelotes, non séché conservé à $-24^{\circ} \mathrm{C}$ ) administrée seulement dans l'expérience $n^{\circ} 1$.

Les cagettes sont maintenues à l'obscurité dans deux étuves de mêmes dimensions et dans les mêmes conditions expérimentales de température $\left(32 \pm 1^{\circ}\right)$. L'humidité relative varie de 45 à $50 \%$.

\section{3. $M E ́ T H O D E$}

Une fois les cagettes remplies d'abeilles ouvrières, elles sont réparties dans deux étuves. Dans l'étuve $\mathbf{A}$, nous avons mis les cagettes d'abeilles témoins et dans l'autre $B$, les cagettes d'abeilles traitées. Chaque jour, avant de sortir les cagettes des étuves pour recevoir les leurres, nous changeons l'emplacement de celles-ci sur les étagères. Nous disposons d'une étagère par groupe de 10 cagettes recevant une même dose de phéromone. Après l'introduction des leurres, nous transférons les cagettes : celles des Abeilles traitées sont mises dans l'étuve A et celles des abeilles témoins, dans l'étuve B. Cet ordre est interverti chaque jour après l'aération des 2 étuves.

Chaque série correspond à 2 lots de 10 cagettes ( 10 cagettes d'abeilles témoins et 10 cagettes d'abeilles traitées) sauf dans l'expérience $n^{\circ} 1$ ( 4 lots de 5 cagettes).

Les leurres sont préparés chaque jour. Pliés, ils sont épinglés sur un support. Les papiers qui servent au lot d'abeilles traitées sont préparés ensemble. Ils reçoivent $0,1 \mathrm{ml}$ d'éthanol à $95^{\circ}$ contenant selon les séries $25,50,75,100,200 \mu \mathrm{g}$ d'acide céto-9 décène- 2 oïque de synthèse. Nous procédons de la même façon pour le lot témoin en préparant les mêmes languettes de papier et en déposant la même quantité d'alcool à $95^{\circ}$ mais sans phéromone.

Les leurres sont présentés chaque matin aux abeilles traitées et témoins pendant 27 jours (exp. $n^{\circ} 1$ ), 19 jours (exp. $\left.n^{\circ} 2\right), 24$ jours (exp. $n^{0} 3$ ).

$\mathrm{Au}$ cours de la série $\mathrm{n}^{0} 1$, nous avons décidé que les deux groupes d'abeilles traitées et témoins seraient répartis de la manière suivante : un lot d'abeilles témoins et un lot d'abeilles traitées recevant un leurre tous les jours, un lot d'abeilles témoins et un lot d'abeilles traitées gardant le même leurre sur lequel on dépose chaque jour la phéromone pendant toute la durée de l'expérience.

Les relevés de mortalité ne commencent que le lendemain de l'encagement.

\section{RÉSULTATS}

Pour toutes les expériences qui vont suivre, nous traçons les courbes de mortalité de chacun des deux lots d'abeilles traitées et témoins à partir des résultats suivants.

Chaque jour, nous notons la mortalité dans chaque cagette. Puis nous faisons la somme des mortalités de chacun des deux lots. Nous cumulons les données dès le deuxième jour et ainsi de suite jusqu'à la fin de l'expérience. Le chiffre final correspond à la somme cumulée de toutes les mortalités durant l'expérience (exp. $n^{\circ} 3$ ). Dans les expériences $n^{\text {os }} 1$ et 2 , les résultats sont interprétés en pourcentages.

\section{Expérience $n^{\circ} 1$}

L'expérience $n^{\circ} 1$ effectuée en mars porte sur 4 lots de 5 cagettes : 2 lots d'abeilles témoins, 2 lots d'abeilles traitées de 50 ouvrières nourries de pollen, de candi et d'eau.

Les ouvrières du lot témoin reçoivent un leurre imprégné d'éthanol $(0,1 \mathrm{ml})$, celles du lot traité, un leurre imprégné d'acide céto- 9 décène- 2 oïque à la dose de $50 \mu \mathrm{g} /$ caget- 
te/jour. Cette série a été préparée de façon à ne renouveler les papiers que dans 2 lots' témoin et traité sur 4 (Fig. 1, Exp. $n^{\circ} 1$ ).

Le premier résultat à signaler est que dans les deux cas, que ce soit les lots à " papiers changés tous les jours " ou les lots à " papiers non changés ", les courbes de pourcentages d'abeilles mortes témoins sont au-dessus de celles d'abeilles mortes traitées. Cela indique que la dose d'acide utilisée est efficace pour au moins une période de 22 jours. La différence la plus marquée entre les 2 courbes se situe au début de l'expérience indiquant par là que ce sont les jeunes ouvrières qui sont les plus sensibles à la
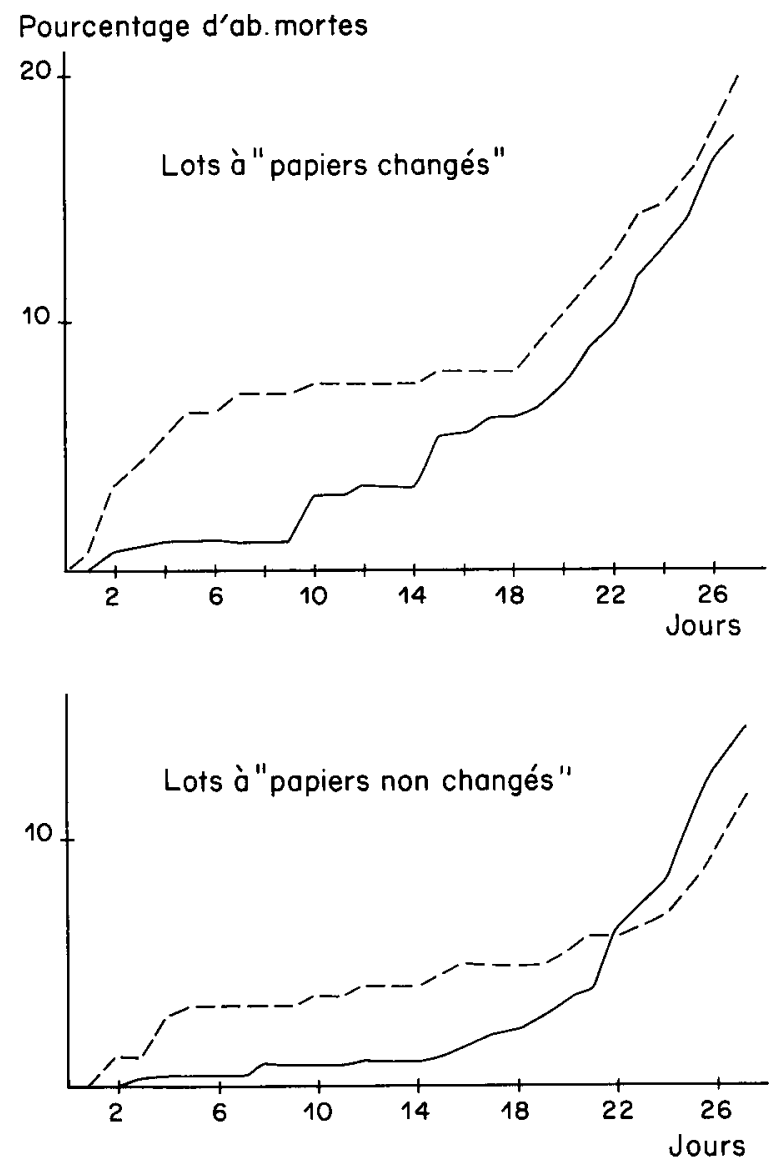

FIG. 1. - Pourcentage d'abeilles mortes dans les lots à "papiers changés" tous les jours et dans les lots à "papiers non changés".

_ lot d'abeilles traitées à la dose de $50 \mu \mathrm{g} / \mathrm{cagette} /$ jour.

- - lot d'abeilles témoins.

FIG. 1. - Percentage of dead bees in groups "baits changed every day " and in groups "baits not changed".

group of honeybees treated with the amount of $50 \mu \mathrm{g} / \mathrm{cage} / \mathrm{day}$. control group. 
présence d'acide céto-9 décène- 2 oïque. C'est dans les lots à " papiers changés " tous les jours que les différences de pourcentage d'abeilles mortes sont les plus importantes.

En fin d'expérience, nous constatons dans les lots à "papiers changés " que le pourcentage de mortalité atteint 20 et $18 \%$ chez les abeilles témoins et traitées alors que dans les lots "papiers non changés ", la mortalité ne dépasse qu'à peine $14 \%$ et $11,5 \%$ chez les abeilles témoins et traitées.

L'analyse des courbes entre les 2 lots d'abeilles traitées et les 2 lots d'abeilles témoins montre bien qu'il y a une action perturbatrice due au fait que les abeilles reçoivent des papiers propres chaque jour. Ces papiers " non familiarisés " introduisent un effet de " stress " qui augmente la mortalité chez les abeilles à " papiers changés ".

On sait que les tarses des pattes des abeilles possèdent des glandes qui laissent sur le substrat une matière volatile odorante (CHAuviN, 1962). D'après nos expériences, il apparait que les papiers " non changés " sur lesquels les abeilles ont marché sont les plus efficaces à réduire la mortalité.

Sur la figure $\mathrm{n}^{\circ} 2$, nous interprétons cet effet de stress par des vagues qui apparaissent par augmentation brusque du taux de mortalité.

Il existe deux vagues que séparent trois périodes de repos de 4 à 5 jours, au $9^{\mathrm{e}}, 14^{\mathrm{e}}$ et $18^{\mathrm{e}}$ jour.

Après cette expérience, nous avons décidé de ne plus mettre de pollen dans les séries suivantes parce que celui-ci prolonge trop la durée de vie. De plus, bien que cet effet perturbateur soit important, nous avons changé quand même les leurres tous les jours pour mieux contrôler les doses d'acide déposées chaque jour sur les leurres.

\section{Expérience $n^{\circ} 2$}

L'expérience $n^{\circ} 2$ effectuée en mai porte sur 4 lots de 10 cagettes : 3 lots d'abeilles traitées et un lot d'abeilles témoins de 50 ouvrières nourries de candi mais ne recevant pas de pollen.

Chaque jour, après avoir retiré les papiers mis la veille, on en remet d'autres dans chacune des cagettes traitées après les avoir imprégnés de $25,50,75 \mu \mathrm{g}$ d'acide céto-9 décène-2 oïque. Les papiers témoins reçoivent la même quantité d'éthanol $(0,1 \mathrm{ml})$ sans acide. Les courbes de mortalité des lots d'abeilles traitées par rapport à la courbe du lot d'abeilles témoins sont présentées sur la figure 3 .

Les courbes de pourcentage d'abeilles mortes ayant reçu 75 et $50 \mu \mathrm{g}$ d'acide céto9 décène-2 oïque sont au-dessus de la courbe des abeilles témoins. Ces deux doses réduisent donc la durée de vie des abeilles traitées surtout après le $10^{\mathrm{e}}$ et le $8^{\mathrm{e}}$ jour. Cependant, nous remarquons une action favorable au début de l'expérience car les ouvrières ne meurent pas avant le $5^{\mathrm{e}}$ et le $7^{\mathrm{e}}$ jour alors que les ouvrières témoins commencent à mourir dès le $1^{\text {er jour. }}$

Seul le lot des abeilles traitées à la dose de $25 \mu \mathrm{g}$ se comporte jusqu'au $10^{\mathrm{e}}$ jour comme le lot des abeilles témoins. Vers le $11^{\mathrm{e}}$ jour, cette dose parait intéressante et aug- 
rourcentage d'ab. mortes
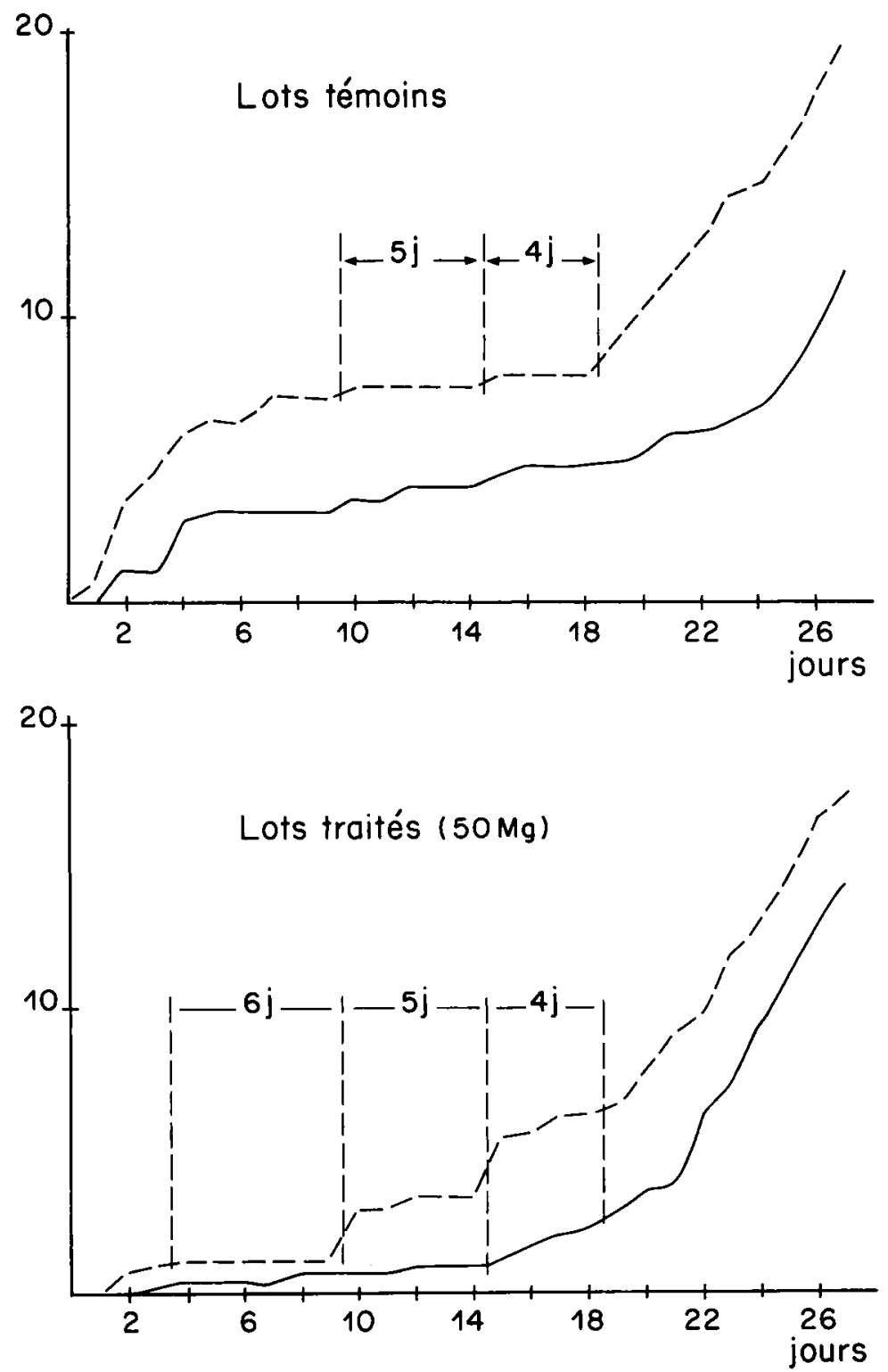

FIG. 2. - Pourcentage d'abeilles mortes dans les lots témoins et traités à la dose de $50 \mu \mathrm{g} /$ cagette/jour.

—_ lot d'abeilles témoins et traitées “ à papiers non changés ».

- _ l lot d'abeilles témoins et traitées à " papiers changés ».

FIG. 2. - Percentage of dead bees in control groups and groups treated with the amount of $50 \mu \mathrm{g} / \mathrm{cage} /$ day. control and treated groups "baits changed ".

- Control and treated groups "baits changed". 


\section{Pourcentage d'ab.mortes}

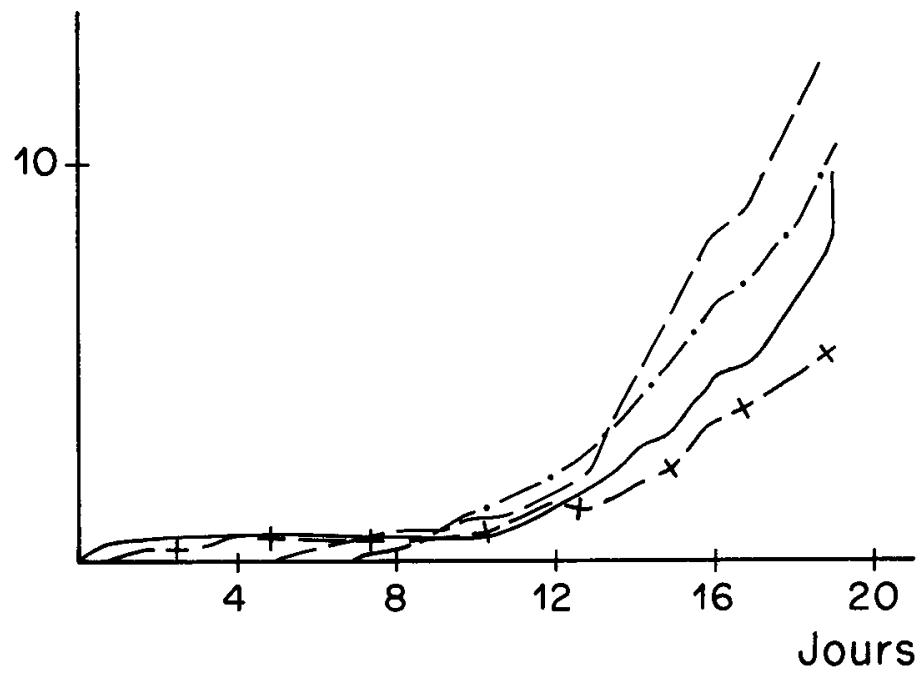

Fig. 3. - Pourcentage d'abeilles mortes ayant reçu pendant 19jours, les doses de 25, 50,75 $\mathrm{\mu g}$ d'acide céto-9 décène-2 ö̈que/cagette/jour.

— lot d'abeilles témoins.

- + lot d'abeilles traitées par $25 \mu \mathrm{g}$.

- - lot d'abeilles traitées par $50 \mu \mathrm{g}$.

lot d'abeilles traitées par $75 \mu \mathrm{g}$.

Fig. 3. - Percentage of dead bees that received the amounts of $25,50,75 \mu \mathrm{g}$ of 9-oxodec-2-enoic acid/cage/day for 19 days.

control group.

group treated with $25 \mu \mathrm{g}$.

group treated with $50 \mu \mathrm{g}$.

group treated with $75 \mu \mathrm{g}$.

mente la durée de vie. A ce moment-là, la courbe des abeilles traitées se situe audessous de celle des abeilles témoins.

\section{Expérience $n^{\circ} 3$}

Dans cette expérience effectuée en juin, nous avons préparé 6 lots de 10 cagettes : 5 lots d'abeilles traitées et un lot d'abeilles témoins de 50 ouvrières nourries comme précédemment (exp. $n^{\circ} 2$ ). Chaque lot d'abeilles traitées reçoit une dose différente d'acide céto- 9 décène- 2 oïque de $25,50,75,100,200 \mu \mathrm{g} /$ cagette/jour.

Les leurres en papier sont changés tous les jours juqu'au $24^{\mathrm{e}}$ jour. L'expérience se poursuit jusqu'au $32^{\mathrm{e}}$ jour mais les abeilles ne reçoivent plus le leurre.

Les résultats sont notés en nombre cumulé d'abeilles mortes (Fig. 4). 
C'est la dose de $25 \mu \mathrm{g}$ d'acide qui parait la plus efficace pour augmenter la survie. Ce résultat confirme l'expérience précédente.

Les doses intermédiaires de 50 et de $75 \mu \mathrm{g}$ sont sans action. Un effet intéressant se manifeste du $14^{\mathrm{e}}$ jour au $25^{\mathrm{e}}$ jour seulement pour la dose de $100 \mu \mathrm{g}$.

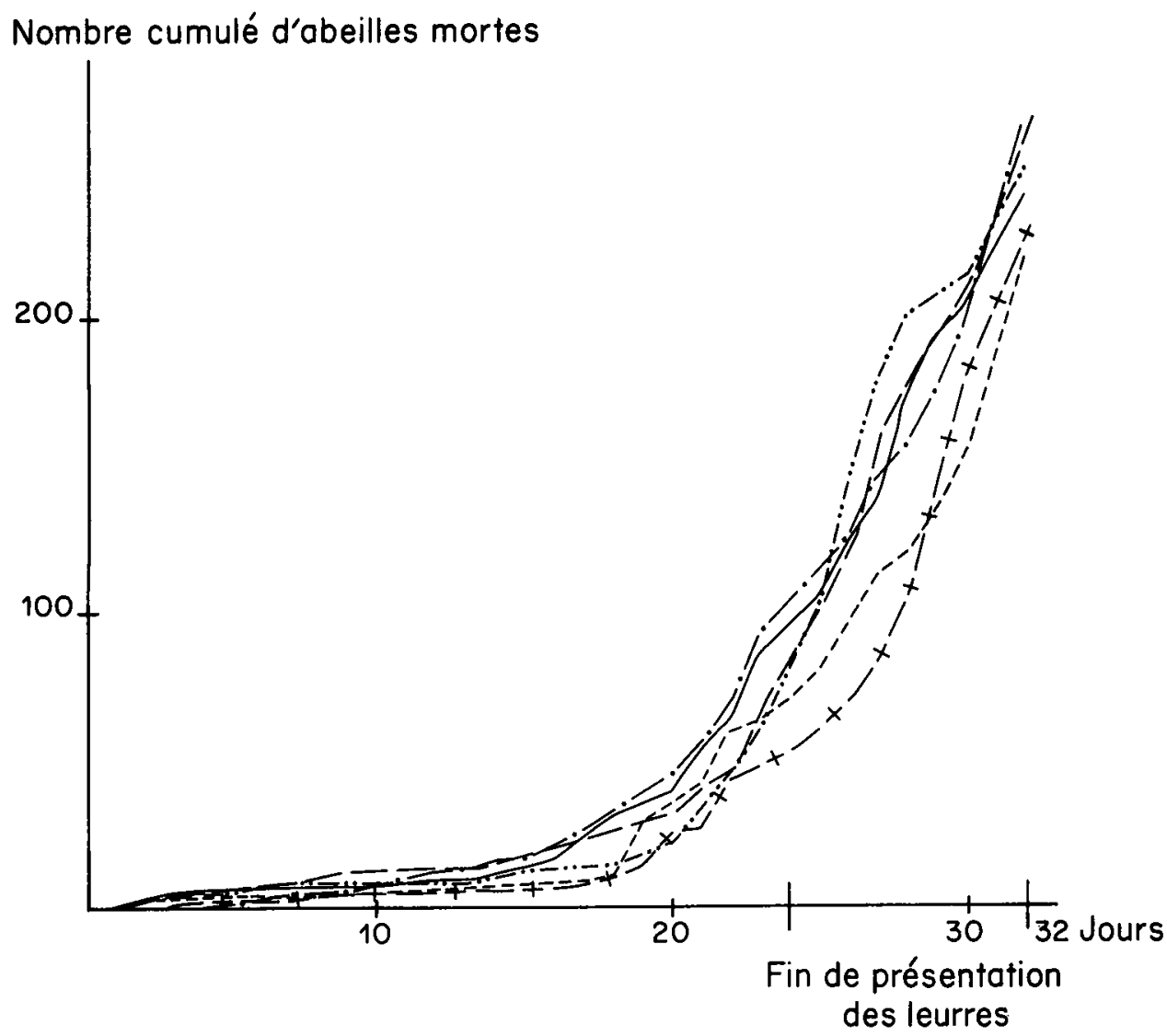

FIG. 4. - Nombre cumulé d'abeilles mortes ayant reçu pendant 24 jours, les doses de $25,50,75,100,200 \mu \mathrm{g}$ d'acide céto-9 décène-2 oïque/cagette/jour.

-...- lot d'abeilles traitées par $100 \mu \mathrm{g}$.

- lot d'abeilles traitées par $75 \mu \mathrm{g}$

-._- lot d'abeilles traitées par $50 \mu \mathrm{g}$.

-+- lot d'abeilles traitées par $25 \mu \mathrm{g}$.

- lot d'abeilles témoins. lot d'abeilles traitées par $200 \mu \mathrm{g}$.

FIG. 4. - Cumulated number of dead bees that received the amounts of $25,50,75,100,200 \mu \mathrm{g}$ of 9-oxodec-2-enoic acid/cage/day for 24 days.

-..-..- group treated with $100 \mu \mathrm{g}$.

- - group treated with $75 \mu \mathrm{g}$.

-. - - group treated with $50 \mu \mathrm{g}$.

-+- group treated with $25 \mu \mathrm{g}$.

control group.

group treated with $200 \mu \mathrm{g}$. 
La dose de $200 \mu \mathrm{g}$ peut être comparée en partie à celle de $25 \mu \mathrm{g}$. Dans les 2 cas, la courbe des abeilles traitées est en dessous de celle des abeilles témoins. L'écart le plus grand entre les 2 courbes se situe :

au $29^{e}$ jour c'est-à-dire à peu près au même moment que celui correspondant à la dose de $25 \mu \mathrm{g}$. Si l'on compare les 2 courbes des abeilles traitées à la dose de 25 et de $200 \mu \mathrm{g}$ d'acide, on s'aperçoit que les ouvrières qui ont reçu $25 \mu \mathrm{g}$ d'acide meurent moins vite à partir de la $2^{\mathrm{e}}$ semaine que celles qui ont reçu $200 \mu \mathrm{g}$. Pour ces deux doses, on peut constater une réduction du taux de la mortalité chez les abeilles traitées.

Pour interpréter les résultats d'une autre façon, nous avons établi les histogrammes suivants (Fig. 5) : pour chaque jour, nous portons en ordonnée les différences absolues obtenues entre les nombres cumulés d'abeilles mortes traitées et le nombre cumulé d'abeilles mortes témoins.

L'analyse des histogrammes montre que seulement les 2 doses de 25 et $200 \mu \mathrm{g}$ d'acide céto- 9 décène- 2 oïque augmentent la durée de vie des abeilles quelle que soit la période de temps considérée. Pour les 3 autres doses de 50, 75, $100 \mu \mathrm{g}$ d'acide, les différences d'abeilles mortes sont tantôt positives tantôt négatives.

D'autre part, nous avons comparé l'amélioration relative de la durée de vie des abeilles traitées à la dose de $25 \mu \mathrm{g}$ d'acide à celle des abeilles témoins. Au bout de 24 jours, cette amélioration est de $45 \%$. D'autres auteurs (Roger, PAIN, 1966) trouvent qu'en présence de jeunes reines vierges, l'amélioration de la durée de vie après le $26^{\mathrm{e}}$ jour est de $60 \%$ (test significatif). Les abeilles témoins sans reine et par conséquent sans acide céto- 9 décène- 2 oïque présentent une durée de vie nettement inférieure. Au cours d'une autre expérience, les mêmes auteurs effectuant des broyats de têtes de jeunes reines fécondes obtiennent une amélioration de la durée de vie de l'ordre de $34,3 \%$ après une période de 28 jours. La dose de $25 \mu \mathrm{g}$ d'acide apporte donc une amélioration de la durée de vie plus grande qu'un broyat de têtes de jeunes reines fécondes.

\section{Comportement des ouvrières à l'égard du leurre phéromoné}

Concernant l'attraction possible des leurres, nous avons surveillé le comportement des abeilles à chaque fois que nous mettions un papier phéromoné. Nous n'avons jamais trouvé 10 abeilles ni même 5 qui soient attirées par les leurres. Par conséquent nos observations confirment les travaux de PAIN (1962), BARBIER, PAIN, 1960* à savoir que cet acide n'est pas attractif. Par contre, ils infirment les travaux de GAvRILOv, SHAPOSHNikova et Trilenko (1975) qui considèrent cette substance comme étant aussi attractive qu'une reine.

* et d'autres auteurs, Butler, 1960. 


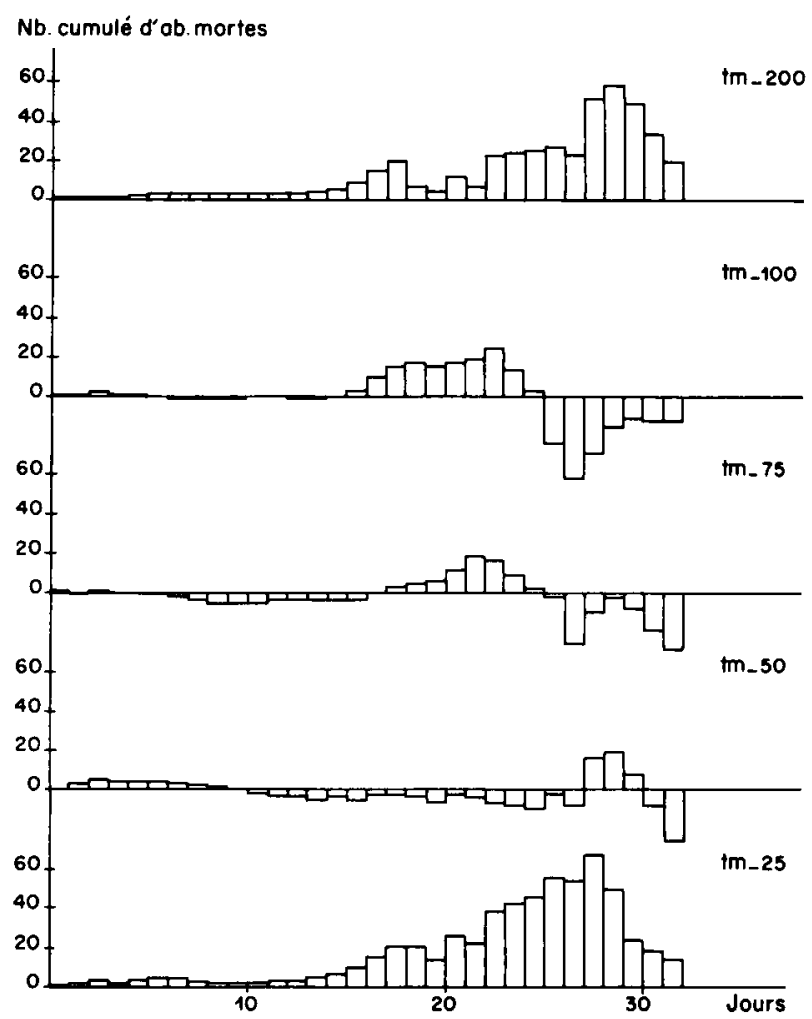

FIG. 5. - Différences absolues entre les nombres cumulés d'abeilles mortes traitées et témoins.

FIG. 5. - Absolute differences between cumulated number of dead control and treated bees.

Bien qu'il n'y ait pas d'attraction au sens défini par PaIN (1961), les abeilles réagissent au papier phéromoné (de $13,5 \mathrm{~cm}^{2}$ de surface) placé dans une cagette de faible volume $\left(468 \mathrm{~m}^{2}\right)$, et peu aéré (vitres pleines). ButLeR et FAIREY signalaient jadis (1963) que la vapeur concentrée d'acide céto- 9 décène- 2 oïque présentait un léger effet inhibiteur sur l'oogenèse. Dans nos essais, bien qu'il n'y ait pas d'attraction vers le leurre, les ouvrières ont réagi favorablement à certaines doses en modifiant leur physiologie.

\section{ANALYSE STATISTIQUE}

Toute la partie statistique ne concerne que l'expérience $n^{0} 3$. Nous avons effectué la comparaison des variances. L'inégalité des variances conduit à la conclusion que les lots sont différents même en dépit de moyennes qui peuvent être égales.

Pour cela, nous avons examiné séparément chaque lot de 10 cagettes et avons calculé un nombre moyen d'abeilles mortes par cagette et par période donnée $\mathrm{X}$ ainsi que 
l'écart-type $S$ autour de cette moyenne. A partir du $21^{\mathrm{c}}$ jour, les valeurs calculées sont les suivantes :

TABL. 1 .

\begin{tabular}{|c|c|c|c|c|c|c|c|c|c|c|c|c|}
\hline & \multicolumn{2}{|c|}{$\tilde{X}^{\text {Témoins }} \quad$ S } & \multicolumn{2}{|c|}{$\overline{\mathrm{X}}^{25 \mu \mathrm{g}} \mathrm{S}$} & \multicolumn{2}{|c|}{$\overline{\mathrm{X}}^{50 \mu \mathrm{g}} \mathrm{S}$} & \multicolumn{2}{|c|}{$\overline{\mathrm{x}}^{75 \mu \mathrm{g}}$} & \multicolumn{2}{|c|}{$100 \mu \mathrm{g}$} & \multicolumn{2}{|c|}{$\overline{\mathrm{X}}^{200 \mu \mathrm{g}} \mathrm{S}$} \\
\hline $\begin{array}{l}21 \mathrm{~J} . \\
24 \mathrm{~J} . \\
30 \mathrm{~J} . \\
32 \mathrm{~J} .\end{array}$ & $\begin{array}{r}5,3 \\
9,6 \\
20,5 \\
24,1\end{array}$ & $\begin{array}{l}2,5 \\
3,69 \\
6,49 \\
6,3\end{array}$ & $\begin{array}{r}2,7 \\
5,3 \\
18,1 \\
22,6\end{array}$ & $\begin{array}{l}2,63 \\
3,47 \\
7,42 \\
8,07\end{array}$ & $\begin{array}{r}5,7 \\
10,4 \\
19,8 \\
26,6\end{array}$ & $\begin{array}{l}5,3 \\
6,55 \\
5,99 \\
7,82\end{array}$ & $\begin{array}{r}4,1 \\
8,3 \\
21,3 \\
26,9\end{array}$ & $\begin{array}{r}1,91 \\
4,00 \\
13,01 \\
11,85\end{array}$ & $\begin{array}{r}3,5 \\
8,2 \\
21,6 \\
25,4\end{array}$ & $\begin{array}{r}2,59 \\
4,89 \\
11,93 \\
11,37\end{array}$ & $\begin{array}{r}4,2 \\
7,3 \\
15,6 \\
22,2\end{array}$ & $\begin{array}{l}5,55 \\
6,04 \\
6,68 \\
6,61\end{array}$ \\
\hline
\end{tabular}

Les valeurs de $\mathrm{F}$ sont établies à partir du rapport $\frac{S^{2} A}{S_{B}^{2}}$ pour $\mathrm{nA}-1$ et $n_{B}-1$. Dans notre cas $n_{A}=n_{B}=10$. La table indique la limite supérieure de $F=3,18$ pour le risque $5 \%$, en fonction du nombre de degrés de liberté : $9+9$. Les valeurs obtenues sont les suivantes :

TABL. 2 .

\begin{tabular}{c|c|c|c|c|c}
\hline \hline & $\mathrm{T}-25$ & $\mathrm{~T}-\mathbf{5 0}$ & $\mathrm{T}-\mathbf{7 5}$ & $\mathrm{T}-100$ & $\mathrm{~T}-200$ \\
\hline $21 \mathrm{~J}$. & 1,11 & 4,49 & 1,71 & 1,08 & 4,95 \\
$24 \mathrm{~J}$. & 1,13 & 3,16 & 1,18 & 1,76 & 2,68 \\
$30 \mathrm{~J}$. & 1,31 & 1,45 & 4,03 & 3,38 & 1,06 \\
$32 \mathrm{~J}$. & 1,64 & 1,54 & 3,54 & 3,26 & 1,10 \\
\hline \hline
\end{tabular}

Pour une même dose et selon la période considérée, on observe l'inégalité des variances, ce qui nous met dans l'impossibilité de traiter les résultats par le test $F$.

La seule série où les variances soient comparables est celle des abeilles traitées par $25 \mu \mathrm{g}$ d'acide céto- 9 décène- 2 oïque. Dans ce cas seulement, la comparaison des moyennes s'avère possible par le test $F$.

Nous avons donc analysé les données en nous basant sur l'écart réduit $\varepsilon$, en effectuant à chaque fois la comparaison de chacun des lots traités avec le lot témoin.

$$
\varepsilon=\frac{P_{A}-P_{B}}{\sqrt{\frac{P_{q}}{n_{A}}+\frac{P_{q}}{n_{B}}}}
$$

$P_{A}$ et $P_{B}$ désignent les pourcentages de mortalités de chaque lot.

$\overline{\mathbf{P}}$ et $\overline{\mathrm{q}}$ désignent les pourcentages évalués sur l'ensemble des lots témoin et traité. $\mathrm{n}_{\mathrm{A}}$ et $\mathrm{n}_{\mathrm{B}}$ sont les effectifs des lots.

Si $\varepsilon \geqslant 1,96$ la différence sera significative. 
L'utilisation de cette méthode nous conduit à prendre comme unité d'étude le lot entier ( 500 abeilles) car le groupe de 50 abeilles (''effectif d'une cagette) s'avère insuffisant étant donné la faible mortalité.

\section{Test de signification}

En fonction des différentes doses administrées et des 4 périodes considérées, le nombre d'abeilles mortes est donné par le tableau suivant :

TABL. 3.

\begin{tabular}{r|c|c|c|c}
\hline \hline & $21 \mathrm{~J}$. & $24 \mathrm{~J}$. & $30 \mathrm{~J}$. & $32 \mathrm{~J}$. \\
\hline témoin & 53 & 96 & 205 & 181 \\
$25 \mu \mathrm{g}$ & 27 & 53 & 198 & 241 \\
$50 \mu \mathrm{g}$ & 57 & 104 & 213 & 266 \\
$75 \mu \mathrm{g}$ & 41 & 83 & 216 & 269 \\
$100 \mu \mathrm{g}$ & 35 & 73 & 156 & 254 \\
$200 \mu \mathrm{g}$ & 42 & & & 222 \\
\hline \hline
\end{tabular}

Pour la période de 21 jours, le calcul de l'écart réduit $\varepsilon$ entre le lot témoin (tm) et les différents lots traités a donné :

\begin{tabular}{c|c|c|c|c}
\hline \hline $\mathrm{tm}-25$ & $\mathrm{tm}-50$ & $\mathrm{tm}-75$ & $\mathrm{tm}-100$ & $\mathrm{tm}-200$ \\
\hline 3,03 & 0,40 & 1,30 & 2,01 & 1,18 \\
\hline \hline
\end{tabular}

Donc, seul le lot traité par $25 \mu \mathrm{g}$ présente une différence significative (sa valeur étant nettement supérieure à 1,96). Le lot traité avec $100 \mu \mathrm{g}$ est à la limite de la différence significative.

Pour la période 24 jours, le calcul de $\varepsilon$ a donné :

\begin{tabular}{c|c|c|c|c}
\hline \hline $\mathrm{tm}-25$ & $\mathrm{tm}-50$ & $\mathrm{tm}-75$ & $\mathrm{tm}-100$ & $\mathrm{tm}-200$ \\
\hline 3,82 & 0,63 & 1,07 & 1,16 & 1,94 \\
\hline \hline
\end{tabular}

Encore une fois le lot traité par $25 \mu \mathrm{g}$ présente une différence significative. Le lot traité par $200 \mu \mathrm{g}$ est à la limite de la signification. De plus, si pour une même période de 24 jours, nous calculons l'écart réduit $\varepsilon$ entre le lot traité par $25 \mu \mathrm{g}$ et tous les autres lots, nous trouvons les résultats suivants :

\begin{tabular}{c|c|c|c|c}
\hline \hline $25-\mathrm{tm}$ & $25-50$ & $25-75$ & $25-100$ & $25-200$ \\
\hline 3,82 & 8,86 & 2,76 & 2,68 & 1,91 \\
\hline
\end{tabular}


Toutes les différences sont significatives. Ceci nous amène à adopter en fait la période de 24 jours comme celle qui renseigne le mieux sur l'efficacité du traitement. En plus, c'est la période limite avant l'arrêt du traitement.

Le calcul de l'écart réduit $\varepsilon$ pour les périodes de 30 et 32 jours montre la perturbation causée par l'arrêt du traitement. Toutes les différences perdent leur signification à l'exception du lot 200 dont la différence reste significative jusqu'au $30^{\mathrm{e}}$ jour $(\varepsilon=3,22)$.

Pour illustrer les résultats, nous avons porté sur la figure 6, l'évolution du pourcentage de mortalité en fonction des périodes de temps considérées pour les différents lots

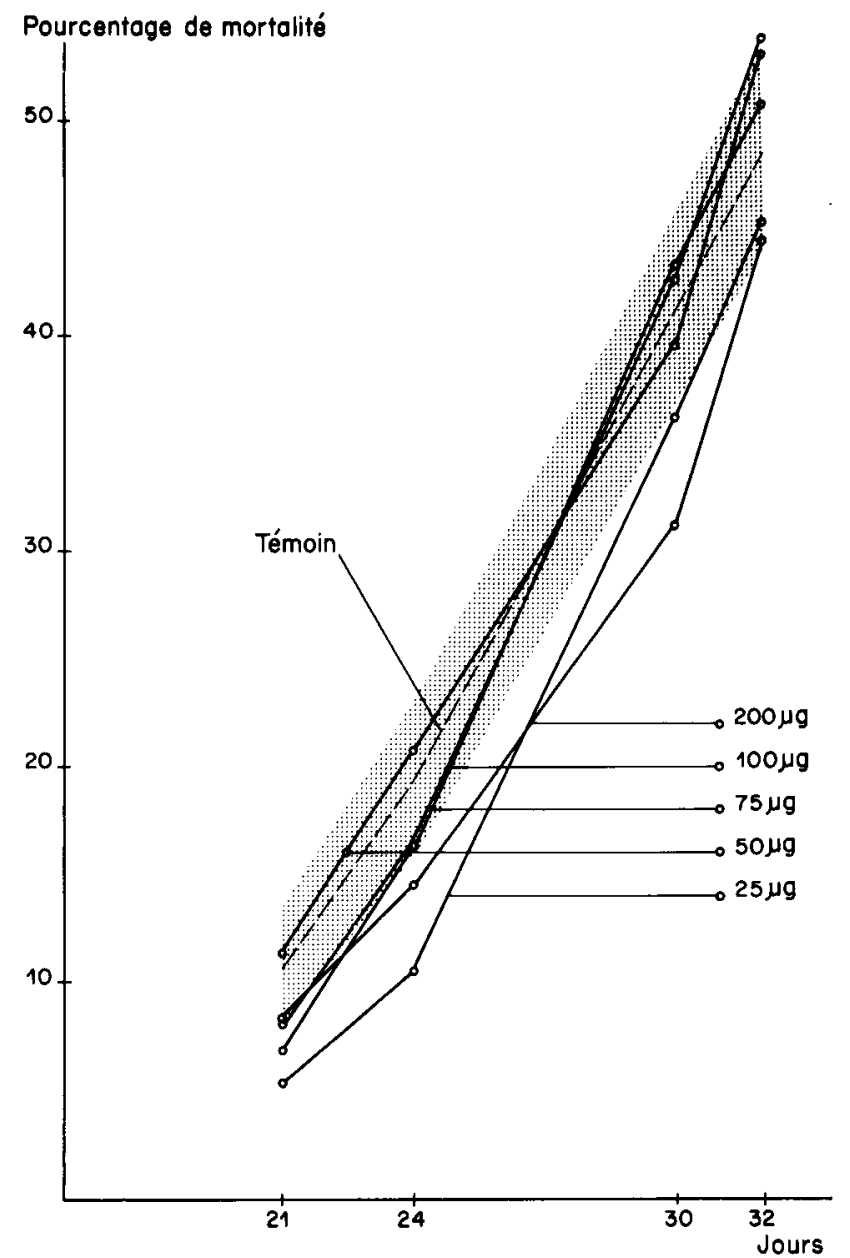

Fig. 6. - Évolution du pourcentage de mortalité en fonction des périodes de temps considérées pour les différents lots traités et le lot témoin avec, en pointillé, les intervalles de confiance du lot témoin.

FIG. 6. - Evolution of the percent mortality with respect to the durations

for the different treated groups and for the control group. In dotted line: confidence intervals for the control group. 
traités ainsi que pour le lot témoin avec, en pointillé, les intervalles de confiance du témoin associés aux valeurs mesurées (en pourcentage).

L'analyse de la figure 6 montre que ce sont les doses de 25 et de $200 \mu \mathrm{g}$ qui sont les plus intéressantes. Pour la dose de $25 \mu \mathrm{g}$, les ouvrières ont une durée de vie nettement améliorée à partir du $21^{\mathrm{e}}$ jour et jusqu'au $25^{\mathrm{e}}$ jour. Pour la dose de $200 \mu \mathrm{g}$, ce n'est qu'après le $24^{\mathrm{e}}$ jour (après l'arrêt du traitement) que les ouvrières réagissent le mieux par rapport aux ouvrières témoins.

Sur la figure 7, nous avons noté l'évolution du pourcentage de mortalité en fonction des doses administrées pour la période de 24 jours avec, en pointillé, les intervalles

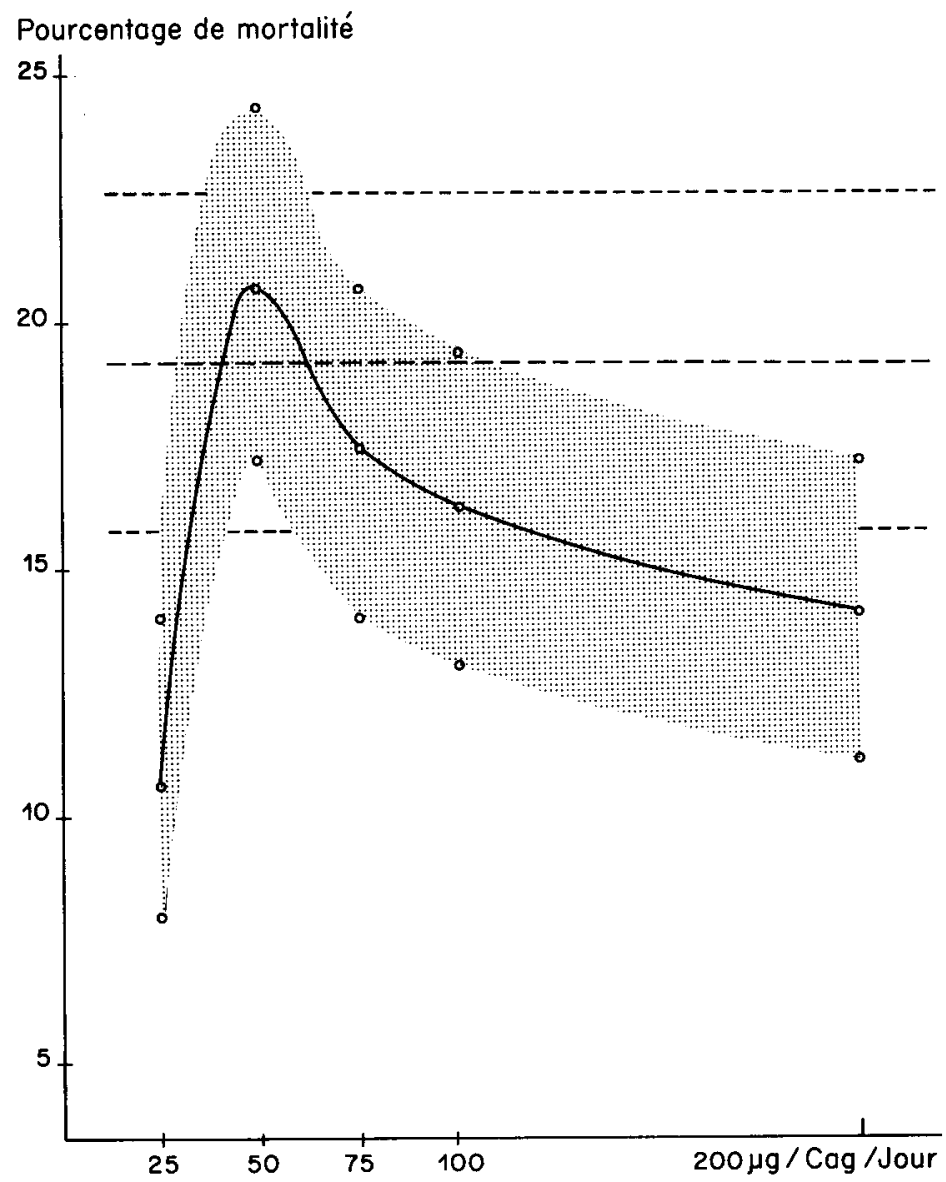

Fig. 7. - Évolution du pourcentage de mortalité en fonction des différentes doses pour la période de 24 jours, avec, en pointillé,

les intervalles de confiance des lots traités et du lot témoin.

FIG. 7. - Evolution of the percent mortality with respect to the different levels for the 24 days duration.

In dotted lines: trust intervals of the treated and control groups. 
de confiance associés aux valeurs mesurées (en pourcentage) chez les abeilles traitées et le témoin. L'intervalle de confiance correspondant à la dose de $25 \mu \mathrm{g}$ se situe en dehors de celui du témoin alors qu'une partie de l'intervalle de confiance de la dose de $200 \mu \mathrm{g}$ se trouve à l'intérieur de celui du témoin.

\section{Hétérogénéité entre cagettes}

Nous l'avons étudié de deux façons :

a) Nous avons dessiné les histogrammes de la figure 8 en portant en abscisse le nombre de cagettes et en ordonnée le nombre cumulé d'abeilles mortes dans chaque cagette sur une période de 24 jours (jour de l'arrêt des traitements).

Nb. cumulé d’ab. mortes
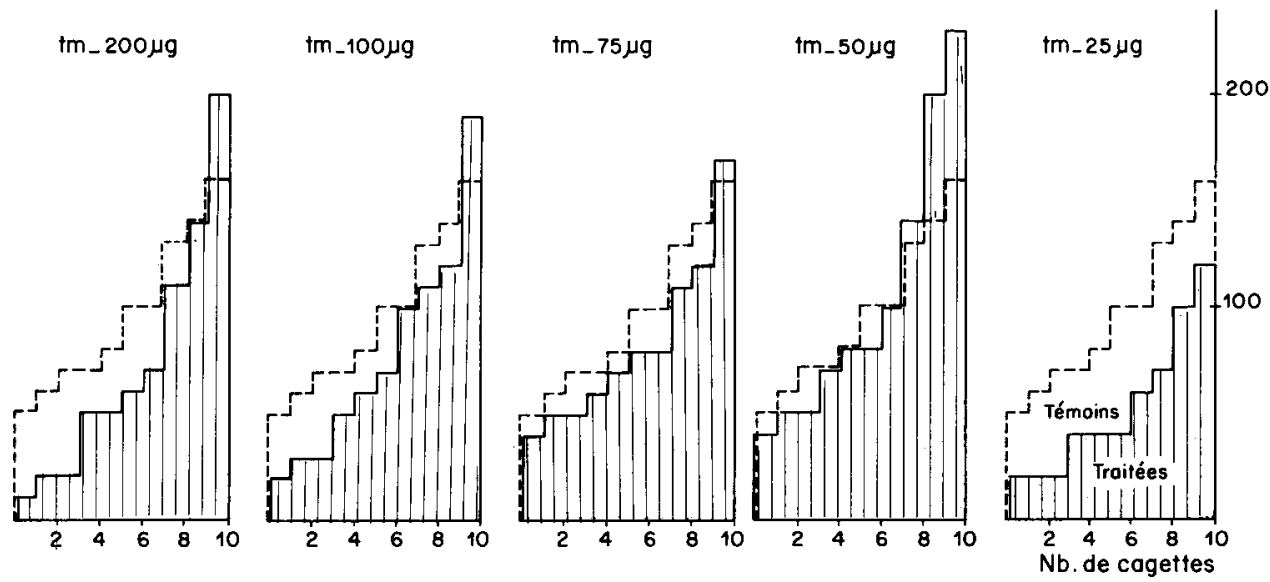

Fig. 8. - Sur une période de 24 jours, mortalité entre les cagettes d'une même série et entre chaque série traitée et la série témoin.

Fig. 8. - Death rate between cages of a same set and between each treated set and the control set, for a 24 days duration.

Les histogrammes mettent en évidence la différence de mortalité d'une part entre les cagettes et d'autre part la différence entre chaque série d'abeilles traitées et la série d'abeilles témoins.

b) Nous avons comparé les pourcentages de mortalité par le test $\mathrm{X}^{2}$. Pour 9 degrés de liberté (10-1 cagettes), la valeur théorique est de 16,919 pour le risque $5 \%$. D'après nos calculs, le pourcentage de mortalité dans les cagettes témoins donne une valeur de $\mathrm{X}^{2}$ égale à 15,87 à peine inférieure. C'est donc seulement dans les cagettes témoins que les différences de mortalité ne sont pas significatives. Dans les cagettes des autres lots contenant les abeilles traitées, les différences de mortalité sont toujours significatives malgré toutes les précautions prises. C'est seulement dans le lot d'abeilles traitées à $25 \mu \mathrm{g}$ d'acide que les différences sont les moins significatives. 
Les abeilles étant des insectes sociaux, ne peuvent vivre isolées. Leur encagement par groupe de 50 montre que chacun de ces groupes se comporte comme s'il était un seul individu. Or normalement, tous les individus ne se comportent pas de la même façon envers un traitement, c'est pour cela qu'on a pour chaque série de cagettes une moyenne et une fluctuation autour de la moyenne qui peut être plus ou moins significative selon le traitement et les conditions expérimentales.

\section{DISCUSSION}

Si nous mettons à part la première expérience réalisée en mars avec des abeilles en provenance de colonies " réactivées " en rucher couvert, on peut observer (exp. $n^{\circ} 2$ et $n^{0} 3$ ) que les courbes de mortalité des abeilles témoins et traitées aux différentes doses d'acide céto- 9 décène- 2 oïque ont la même allure jusqu'au $10^{e}$ et $14^{e}$ jour de vie en cagette : le nombre d'ouvrières mortes reste le même.

Ce n'est qu'à partir du $14^{\mathrm{e}}$ jour que les abeilles traitées réagissent plus ou moins favorablement selon les quantités d'acide céto-9 décène- 2 oïque déposées chaque jour sur les leurres.

L'effet positif de l'acide sur la survie des ouvrières est donc progressif. RoGER, PAIN (1966) avaient déjà noté que l'influence bénéfique des reines ne se fait sentir qu'après plus de 15 jours d'encagement des ouvrières auprès des reines.

Après l'arrêt des traitements (le $25^{\mathrm{e}}$ jour) et jusqu'à la fin des expériences (le $32^{\mathrm{e}}$. jour), on remarque que les ouvrières réagissent différemment selon les doses qu'elles ont reçu au préalable :

- Aux doses de 50, 75, $100 \mu \mathrm{g}$ d'acide, l'amélioration de la survie est inexistante.

- A la dose de $25 \mu \mathrm{g}$, l'amélioration positive constatée à partir du 14e jour s'atténue en fin d'expérience.

- Par contre, à la dose de $200 \mu \mathrm{g}$, l'effet est intensifié en fin d'expérience.

C'est ce que traduit l'analyse statistique. Le calcul de l'écart réduit met bien en évidence la perturbation causée par l'arrêt des traitements sauf pour le lot d'abeilles traitées à $200 \mu \mathrm{g}$ d'acide.

C'est donc la dose de $25 \mu \mathrm{g}$ qui optimise la durée de vie des ouvrières encagées. C'est aussi cette dose qui agit sur le butinage (EMEILlat, 1977). Toutefois, la dose de $200 \mu \mathrm{g}$ d'acide reste intéressante.

Pour expliquer l'action presque comparable de ces deux doses de phéromone royale (acide céto- 9 décène- 2 trans oïque) dont l'une est presque 10 fois plus forte que l'autre, nous émettons l'hypothèse que cette phéromone dont les activités sont multiples agit selon deux mécanismes que nous nous proposons d'examiner par la suite.

Aux doses faibles (moins de 25 à $25 \mu \mathrm{g}$ ), l'effet serait d'ordre sensoriel de type psychosomatique : le papier phéromoné remplaçant en partie la reine. 
Aux doses fortes (à partir de $200 \mu \mathrm{g}$ ), l'effet serait d'ordre pharmacodynamique : la phéromone agissant par imprégnation biochimique de masse, les récepteurs sensoriels étant bloqués.

Entre ces deux doses, l'absence d'effet pourrait s'expliquer par une saturation plus ou moins importante des récepteurs sensoriels.

Ces résultats suggèrent la poursuite d'autres expériences qui permettront de définir jusqu'à quelle dose on peut descendre pour obtenir un effet positif sur la survie et les modes d'action de cette phéromone.

\section{CONCLUSION}

L'analyse des courbes de mortalité des abeilles des séries traitées par rapport aux abeilles des séries témoins nous montre que les doses qui augmentent la durée de vie des abeilles encagées sont les doses de 25 et de $200 \mu \mathrm{g}$ d'acide céto- 9 décène- 2 oïque de synthèse (Fig. 3 et 4 ).

Mais c'est la dose de $25 \mu \mathrm{g}$ d'acide qui augmente de façon significative la durée de vie des ouvrières surtout à partir du $21^{\mathrm{e}}$ jour et jusqu'au $25^{\mathrm{e}}$ jour de leur captivité (Fig. 4). C'est aussi à cette dose que les différences de mortalité entre cagettes sont les moins significatives (Fig. 8).

Le pourcentage de mortalité en fonction de cette dose pour la période de 24 jours se situe avec son intervalle de confiance en dehors de celui du témoin (Fig. 7).

La dose de $200 \mu \mathrm{g}$ d'acide a une action déjà moins efficace. Cette action se fait sentir plus tardivement, après le $24^{e}$ jour c'est-à-dire après l'arrêt du traitement (Fig. 4).

Le pourcentage de mortalité en fonction de cette dose pour la période de 24 jours se situe en partie seulement en dehors de l'intervalle de confiance du témoin (Fig. 7).

Les doses intermédiaires de 50,75 et $100 \mu \mathrm{g}$ d'acide céto- 9 décène- 2 oïque n'améliorent pas la durée de vie des ouvrières encagées (Fig. 5, 6, 7).

Nous pouvons donc conclure que la dose de $25 \mu \mathrm{g}$ d'acide est la plus favorable. Elle présente une efficacité plus grande qu'une reine de substitution imprégnée d'extraits de têtes de jeunes reines fécondes. Toutefois, cette dose n'augmente pas la durée de vie des ouvrières captives comme le ferait une jeune reine vierge.

L'hypothèse de deux mécanismes d'action est suggérée.

Reçu pour publication en janvier 1981.

Eingegangen im Januar 1981 .

\section{ZUSAMMENFASSUNG}

EFFEKT DER 9-KETO-2 TRANS-DECENSÄURE (PHEROMON DER BIENENKÖNIGIN APIS MELLIFICA L.) AUF DIE MORTALITÄTSRATE GEK ÄFIGTER ARBEITSBIENEN

Im Verlaufe früherer Untersuchungen (Roger und PAIN, 1966) hat sich gezeigt, dass Königinnen (lebend, tot oder zerquetscht) einen günstigen Einfluss auf die Mortalitätsrate der Begleitbienen ausüben. 
Ausgehend von der Tatsache, dass die Königinnen 9-Keto-2 trans-Decensäure enthalten, wurde geprüft, ob diese Säure nicht zum Teil für die Verringerung der Mortalität von kleinen Gruppen von Arbeiterinnen bekannten Alters verantwortlich ist.

Für diese Untersuchung haben wir 50 Jungbienen im Alter von ein bis drei Tagen von schlüpfenden Brutwaben gekäfigt. Diese Bienen wurden mit Zuckerteig (Honig und Zuckerguss $1: 3$ ), aber nicht mit Pollen gefüttert - ausser in Versuch 1.

Die Königinnen wurden durch Papierköder (Chromatographierpapier) ersetzt, die aus Streifen von $15 \mathrm{~mm}$ Breite und $90 \mathrm{~mm}$ Länge vorbereitet waren. Die Köder wurden täglich mit $0,1 \mathrm{ml}$ Äthanol $95 \%$ imprägniert, in dem je nach Versuchsserie 25, 50, 75, 100 und $200 \mu \mathrm{g}$ 9-Keto-2 trans-Decensäure je Käfig enthalten war.

Die Kontrollsansätze wurden in derselben Weise behandelt, indem sie mit $95 \%$ Alkohol imprägnierte Papierstreifen - jedoch ohne Pheromon - enthielten. Die Papierstreifen wurden durch eine Öffnung im Deckel eingeführt und mittels eines Korkpfropfens fixiert.

Die Käfige wurden im Dunklen in zwei Brutschränken gleicher Dimension, unter denselben experimentellen Bedingungen von Temperatur $\left(32 \pm 1^{\circ}\right)$ und relativer Feuchte (45-50\%) gehalten.

Täglich wurden die Käfige mit den Kontrollbienen, bzw. die Käfige mit den behandelten Bienen, die sich in dem einen der Brutschränke befanden, in den anderen verstellt und umgekehrt.

Jede Serie besteht aus Gruppen zu je $10 \mathrm{Käfigen} \mathrm{(Versuch} \mathrm{Nr.} \mathrm{2:} 4$ Gruppen einschliesslich einer Kontrolle, Versuch Nr. 3: 6 Gruppen einschliesslich einer Kontrolle) ausser dem Versuch Nr. 1, der 4 Gruppen zu 5 Käfigen umfasste, von denen 2 Kontrollen waren.

Die Attrappen wurden jeden Morgen dargeboten, und zwar während 27 Tagen (Versuch Nr. 1), 19 Tagen (Versuch Nr. 2) und 24 Tagen (Versuch Nr. 3).

Mit der Entnahme der toten Bienen wurde am Tag nach der Füllung der Käfige begonnen. Jeden Tag haben wir die Mortalität in jedem Käfig notiert. Dann haben wir die Summe der Mortalität von jeder von zwei Gruppen gebildet. Vom 2. Tag an haben wir die Daten aufsummiert und ebenso in der Folge bis zum Ende des Versuches. Die Endzahl entspricht der kumulierten Summe sämtlicher Mortalitäten während des Versuches (Versuch Nr. 3). In den Versuchen 1 und 2 sind die Ergebnisse in Prozenten ausgedrückt.

Die Analyse der Mortalitätskurven der behandelten Serien im Vergleich zu den Kontrollserien zeigt uns, dass nur die beiden Dosen $25 \mu \mathrm{g}$ und $200 \mu \mathrm{g}$ synthetischer 9-Keto-2 trans-Decensäure das Leben der gekäfigten Bienen verlängert (Abb. 3 und 4). Aber nur die Dosis von $25 \mu \mathrm{g}$ der Säure erhöht die Lebensdauer der Arbeiterinnen in signifikanter Weise, besonders in der Periode vom 21. bis zum 25. Tag der Käfighaltung (Abb. 4). Bei dieser Dosis sind auch die Mortalitätsunterschiede zwischen den Käfigen am wenigsten signifikant (Abb. 8). Der Prozentsatz der Mortalität für die Periode von 24 Tagen in Funktion dieser Dosis liegt mit seinem Vertrauensbereich ausserhalb desjenigen der Kontrolle (Abb. 7). Die Dosis von $200 \mu \mathrm{g}$ der Säure hat schon eine geringere Wirkung. Diese Wirkung tritt erst spät in Erscheinung, erst nach dem 24. Tag, d.h. erst nach Beendigung der Behandlung (Abb. 4). Der Prozentsatz der Mortalität für die Periode von 24 Tagen, in Funktion dieser Dosis liegt teilweise ausserhalb des Vertrauensbereiches der Kontrolle (Abb. 7). Die mittleren Dosen von 50, 75 und $100 \mu \mathrm{g}$ 9-Keto- 2 transDecensäure führten zu keiner Verlängerung der Lebensdauer der gekäfigten Bienen (Abb. 5, 6, 7).

Wir können daraus den Schluss ziehen, dass die Dosis von $25 \mu \mathrm{g}$ die günstigste ist. Sie übt eine stärkere Wirkung aus als eine Ersatzkönigin, die mit Extrakten aus den Köpfen junger begatteter Königinnen imprägniert wurde. Aber diese Dosis erreicht nicht die Verlängerung der Lebensdauer gekäfigter Arbeitsbienen, wie sie eine junge unbegattete Königin bewirkt.

So wird eine Hypothese von zwei Wirkungsmechanismen vorgeschlagen.

\section{SUMMARY}

\section{INFLUENCE OF 9-OXODEC-2-ENOIC ACID (APIS MELLIFICA QUEEN PHEROMONE) ON DEATH RATE OF CAGED WORKER BEES}

Earlier expriments by ROGER and PAIN (1966) mentioned the beneficial influence of queen bees (alive, dead, crushed) on death rate of the attendant workers. Since honey bee quees contain 9-oxodec-2-enoic 
acid, it was verified whether this acid would not be partly responsible for the mortality decrease of small groups of workers whose age is known.

For this study 50 1-3 days old newly emerged workers from brood frames were caged and fed candy (water and icing sugar $1: 3$ ) but no pollen, except in experiment $n^{\circ} 1$. Paper baits in the form of small strips $15 \mathrm{~mm}$ wide and $90 \mathrm{~mm}$ long were put in the place of queens. They were impregnated daily with $0,1 \mathrm{ml} 95^{\circ}$ ethanol and $25,50,75,100$ and $200 \mu \mathrm{g}$ of synthetic 9-oxodec-2-enoic acid per cage depending on the experiments. The same was done for control groups which received paper baits impregnated with $0,1 \mathrm{ml} 95^{\circ}$ ethanol but without pheromone. Papers were introduced through one of the openings of the $1^{\circ}$ ) and relative humidity (45-50\%) conditions.

Every day control and treated cages of honeybees kept in one of the incubator were moved in the other and vice versa. Every set included groups of 10 cages (experiment No. $3: 6$ groups, control group included), except in experiment No. 1 (4 groups of 5 cages, 2 control cages included). Baits were given every morning for 27 days (experiment $\mathrm{N}^{\circ} 1$ ), 19 days (experiment $\mathrm{N}^{\circ} 2$ ) and 24 days (experiment $\mathrm{N}^{\circ} .3$ ).

Mortality records began the day after the bees were caged and continued daily for each cage. The mortality of both groups were cumulated together beginning with the second day and so on till the end of experiment. The final number was the cumulated sum of all deaths during the experiment (experiment No. 3). In experiments Nos. 1 and 2 results were given in percentages.

Analysis of the mortality of honeybees from treated and control groups shows that the amounts of synthetic 9-oxodec-2-enoic acid increased the survival of caged honeybees at 25 and $200 \mu \mathrm{g}$ (Fig. 3 and 4). The increase in survival of workers fed $25 \mu \mathrm{g}$ of acid was significant especially from the 21 st to the 25 th day of their captivity (Fig. 4). At this level the differences of deaths between cages are least significant (Fig. 8). The percent mortality for this level during the period of 24 days is situated within the confidence limits of the control (Fig. 7).

The result of feeding $200 \mu \mathrm{g}$ of acid is less effective. This effect is felt later, after the 24 th day, that is after the treatment is stopped (Fig. 4). The percentage of death in terms of this amount for the period of 24 days is only partly located outside the confidence limits of the control (Fig. 7).

Intermediate amounts of 50,75 and $100 \mu \mathrm{g}$ of 9-oxodec-2-enoic acid do not improve survival of caged honey bees (Fig. 5, 6, 7). Therefore we can only conclude that $25 \mu \mathrm{g}$ is the most propitions amount. Its efficiency is greater than that of a queen qubstitute impregnated with extracts from young mated queens. Nevertheless this amount does not increase survival of caged workers as a young virgin queen would.

The hypothesis for both mechanisms of action is suggested.

\section{BIBLIOGRAPHIE}

Barbier M., Lederer E., Reichstein T., Schindler O., 1960. - Auftrennung der sauren Anteile von Extrakten aus Bienköniginnen (Apis mellifica L.); Isolierung des als Königinnen-Substanz bezeichneten Pheromones. Helv. Chim. Acta, 43, 1682-1683.

BARBIER M., PAin J., 1960. - Étude de la sécrétion des glandes mandibulaires des reines et des ouvrières d'abeilles (Apis mellifica) par chromatographie en phase gazeuse. C.R. Acad. Sci., Fr., 250, 37403742.

BUTLER C. G., 1960. - Queen Recognition by Worker honeybees (Apis mellifera L.). Experientia, 16, 424426.

Butler C. G., Fairey E. M., 1963. - The role of the queen in preventing oogenesis in worker honeybees. J. Apicult. Res., G.B., 2 (1), 14-18.

BUTLER C.G., Simpson J., 1958. - The source of the queen substance of the honey-bee (Apis mellifera L.). Proc. R. Ent. Soc. Lond. (A), 33, 7-9.

Callow R. K., Johnston N. C., 1960. - The Chemical Constitution and Synthesis of Queen Substance of Honey bees (Apis mellifera L.). Bee World, G.B., 41, 152-153. 
Chauvin R., 1962. - Sur l'épagine $\varepsilon$ et sur les glandes tarsales d'Arnhart. Insectes Soc., Fr., 9 (1), 1-5.

EMEILLAT R., 1977. - Le comportement des ouvrières d'abeille (Apis mellifica L.) et les phéromones de la reine. Mémoire de fin d'étude. Station de Recherches sur l'Abeille et les Insectes Sociaux. 91440 Bures sur Yvette. 47 pages.

Gavrilov B. N., Shaposhnikova N. G., Trilenko V. A., 1975. - Étude de l'activité biologique de la phéromone synthétique de la reine d'abeilles, l'acide céto-9 décène-2 oïque. $S b$. Rab. Leningrad Vet. Inst. 40 (en russe) traduction française I.N.R.A. Versailles.

PAIN J., 1961. - Sur la phéromone des reines d'abeilles et ses effets physiologiques. Thèse Sc. Nat., Paris, $\mathrm{n}^{\circ} 4526,103$ pages.

Pain J., 1962. - L'importance du pouvoir d'attraction des reines d'abeilles pour le prélèvement de la phéromone. Symp. gen. et Biol. Italica, 10 (4 Congrès U.I.E.I.S. Pavie 1961), 189-197.

PAIN J., 1966. - Nouveau modèle de cages expérimentales pour le maintien d'abeilles en captivité. Ann. Abeille, 9 (1), 71-76.

RenNer M., VierLing G., 1977. - Die Rolle des Taschendrüsenpheromons beim Hochzeitsflug der Bienen-Konigin. Behav. Ecol. Sociol., (R.F.A.), 2 (3), 329-338.

Roger B., Pain J., 1966. - L'influence de la Reine d'abeilles (Apis mellifica L.) sur le taux de mortalité des ouvrières accompagnatrices. Ann Abeille, 9 (1), 5-36.

Roger B., 1971. - L'influence de la Reine d'abeilles (Apis mellifica L.) sur la prise de nourritures des ouvrières accompagnatrices. Apidologie, 2 (2), 123-155. 